\title{
Surgical Excision of Placenta with Lower Uterine Segment as a Conservative Management in a Case of Placenta Accreta: A Case Report
}

\author{
Ahmed Sherif Abdel Hamid Abdel Wahab ${ }^{1 *}$, Mohamed Mohamed Yaseen² \\ ${ }^{1}$ Department of Obstetrics \& Gynecology, Ain-Shams University, Cairo, Egypt \\ ${ }^{2}$ Department of Urology, Ain-Shams University, Cairo, Egypt \\ Email: *ahmedgyna@yahoo.com
}

How to cite this paper: Wahab, A.S.A.H.A. and Yaseen, M.M. (2018) Surgical Excision of Placenta with Lower Uterine Segment as a Conservative Management in a Case of Placenta Accreta: A Case Report. Open Journal of Obstetrics and Gynecology, 8, 63-68.

https://doi.org/10.4236/ojog.2018.81008

Received: December 30, 2017

Accepted: January 22, 2018

Published: January 25, 2018

Copyright $\odot 2018$ by authors and Scientific Research Publishing Inc. This work is licensed under the Creative Commons Attribution International License (CC BY 4.0).

http://creativecommons.org/licenses/by/4.0/

\begin{abstract}
Introduction: Placenta accreta is due to invasive placental implantation. It is diagnosed when there is failure of delivery of a retained placenta. This is usually complicated by massive intrapartum hemorrhage that ends by hysterectomy. Case: We report a case of conservative management in a case of placenta accreta involving an elliptical shape incision of the lower segment with removal of placenta with underlying lower uterine segment in a stable patient desiring future fertility. Conclusion: Conservative management may be valid in carefully selected cases of placenta accreta diagnosed pre-operatively in tertiary hospitals with availability of blood-bank and multi-disciplinary approach.
\end{abstract}

\section{Keywords}

Placenta Accreta, Conservative Management, Elliptical Incision, Cystostomy

\section{Introduction}

Placenta accreta is a serious complication associated with high maternal morbidity and mortality. The invasive placental implantation is usually caused by a defect in the decidua basalis [1]. Predisposing factors include previous caesarean sections, previous uterine scars, and prior uterine curettage [2]. Other risk factors associated with placenta accreta are maternal age over 35 years; multiparity ( $>4$ pregnancies); previous placenta previa; prior intrauterine infections and low decidual natural killer cells [2] [3] [4]. Placenta accreta is diagnosed 
histologically when there is trophoblastic invasion of the myometrium without intervening decidua [1]. The different types of morbid adherent placenta include the accreta when the invasion is limited to the superficial myometrium, the second type is placenta increta when invasion extends into deeper myometrium, the last type is placenta percreta when invasion extends through the serosa and/or adjacent pelvic organs [5].

The diagnosis is usually achieved antenatally in high-risk pregnancies by the use of ultrasound and doppler [1]. This helps in taking the appropriate plan to reduce maternal morbidity and mortality. Accidentally, most cases are diagnosed only at the time of operation when forcible unsuccessful attempts at manual removal of the placenta [6]. Severe hemorrhage may result and may lead to complications such as shock; DIC; acute renal failure; massive transfusion of blood products; ARDS; loss of fertility due to hysterectomy [1]. Mortality is as high as $7 \%[7]$.

Usually, caesarean hysterectomy has been the preferred management in cases for placenta accreta [1]. Not only does caesarean hysterectomy preclude future fertility, but it is associated with significant perioperative risks [7].

The aim of the current case report is to report a new surgical technique that succeeded in uterine conservation in cases of placenta accreta.

\section{Case}

A 35 years old Para 2 previous 2 cesarean section patient diagnosed as placenta accreta by sonar and Doppler (Figure 1), presented at 36 weeks with an attack of antepartum hemorrhage in a big private hospital (This was the first pregnancy from the second marriage) and the patient wanted to preserve the uterus; therefore, conservative surgery was done. Consent of hysterectomy and bladder opening were taken from the patient in case of failure of conservative surgery. Four units of packed RBCs and Four units of Fresh frozen plasma were prepared and cross matched.The preoperative hemoglobin of the patient was $10 \mathrm{gm} / \mathrm{dl}$.

Technique: Through a mid-line infra-umbilical incision the anterior abdominal

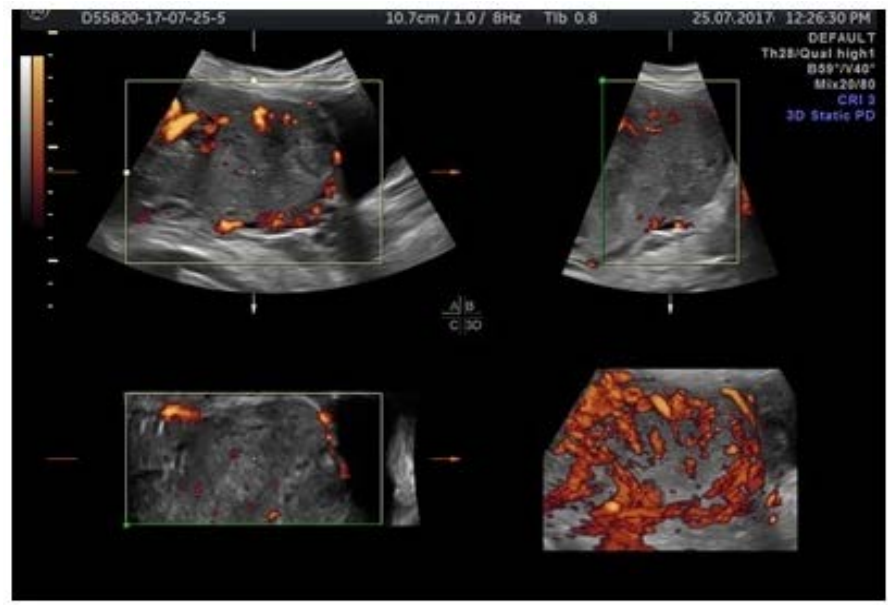

Figure 1. Doppler ultrasound of the placenta accreta of the patient. 
wall was opened in layers. The urologist opened the urinary bladder through a longitudinal incision at the doom and its posterior wall was grasped by Alice forceps followed by downwards dissection of the bladder until the cervix, the idea of cystostomy was to avoid bladder injury in further steps and to place 2 ureteric catheters. Through a mid-line incision the upper uterine segment was incised with delivery of a single living female baby. Ecbolics were given for the patient in the form of oxytocin 20 IU IV, carbetocin 100 microgram IV in a single dose. The placenta failed to be detached from the uterus. Bilateral uterine artery ligation was done. The uterine incision was extended to be elliptical in shape with its lower end at the cervix (Figure 2). The placenta was removed by attached lower uterine segment. The lower part of the incision was closed by Vicryl 1 suture in one layer locked sawing suture manner while the upper thicker part was closed in continuous 2 layers. Bilateral internal iliac artery ligation was done to insure haemostasis. The urologist placed 2 ureteric catheters and then closed the urinary bladder by Vicryl 2/0 suture in 2 layers. An omental flap was placed between the bladder and the uterus. The patient took 2 units of packed RBCs and 2 units of fresh frozen plasma intraoperative.

An indwelling urinary catheter was left to drain the urinary bladder. An intra-peritoneal drain was inserted. Post operative $\mathrm{HB}$ was $8 \mathrm{gm} / \mathrm{dl}$ so another 2 units of packed RBCs were given; the intraperitoneal drain was removed after 48 hours. The urinary catheter was removed after one week. The ureteric catheters were removed by cystoscope after 3 weeks. The patient made an uneventful

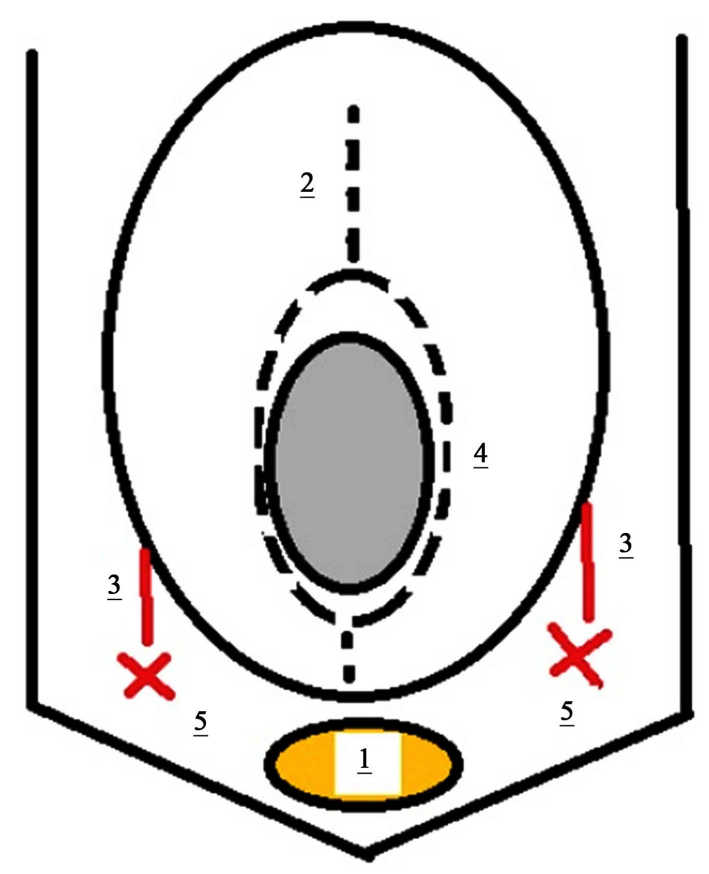

Figure 2. Illustrating figure showing idea of technique; 1-Dissection of the bladder downward beneath the cervix. 2-Upper segment incision to deliver the fetus. 3-Bilateral uterine artery ligation. 4-elliptical incision to involve the placenta (then closure not illustrated in figure). 5-Bilateral internal iliac artery ligation. 
recovery. Follow up of the patient after 6 weeks revealed normal involuted uterus. The patient regained menstruation after 14 weeks. The Patient gave her consent for the case report to be published without mentioning her name.

\section{Discussion}

The incidence of placenta accreta reaches $1 / 1000$ deliveries due to the increase in caesarean section rate [1].

Traditionally, caesarean hysterectomy has been the preferred method of management especially after failure of placental detachment for fear of massive hemorrhage. Caesarean hysterectomy is a major operation that could cause significant maternal morbidity and mortality. Therefore in the current case the urinary bladder was deliberately opened and carefully dissected before performing the conservative approach in order to facilitate sound repair at the end of the operation, in addition to, bilateral uterine artery ligation before the uterine incision and bilateral internal artery ligation after closure of the incision in order to decrease blood loss associated with this surgical approach.

This approach involves elliptical shape incision of the lower uterine segment providing that the patient remains hemodynamic stable, without life-threatening hemorrhage, and with a desire for ongoing fertility. Our approach succeeded because there was enough lower uterine segment after excision of the placenta to be repaired, in another cases the uterine defect must be evaluated carefully to apply such technique.

Several forms of conservative solutions of placenta accreta have been reported in the literature. They vary from partial resection of the placental bed and postoperative administration of methotrexate. Most of these cases are followed by delayed hysterectomy [8].

Another case report by Gaspare et al., 2014 [9] they first bilateral uterine artery ligation then they removed the placenta after which they ligated the internal iliac arteries and placed intrauterine Bakri balloon tamponade. They concluded that the application of the Bakri balloon and pelvic devasculariztion to these patients decreases the need for hysterectomy and the patient's fertility is protected.

Our approach succeeded due as there was enough lower uterine segment after excision of the placenta to be repaired, in another cases the uterine defect must be evaluated carefully to apply such technique.

In the study of Palacios Jaraquemda et al., 2004 [10] they removed the placenta with underlying myometrium and the anterior uterine defect was repaired by the use of primary pulley sutures, polyglycolic mesh fixation outside the primary suture and fibrin glue. Finally they applied non adherent cellulose layer over that reconstruction. They reported good success with the use of this technique to conserve the uterus.

Another technique to preserve the uterus is the Triple P Procedure described by Chandraharan et al., 2012 [11]. It involved 3 P steps. Perioperative placental localization was performed by the use of ultrasound and MRI, the delivery of the 
fetus was achieved by transverse uterine incision. Pelvic devascularization was done by occlusive ballons inserted in internal iliac arteries perioperative by intervention radiology team. Placental non separation was achieved with myometrial excision and then reconstruction of uterine wall. The difference between our technique and their technique is that we used longitudinal upper segment incision and the elliptical extension was in the same line of original incision that aided in the closure of the uterine defect after the removal of the placenta.

\section{Conclusion}

Our approach depended on intentionally doing cystostomy to allow good dissection of bladder and placing of ureteric catheters. The uterine incision was longitudinal in shape to deliver fetus then bilateral uterine artery ligation. Next step was elliptical incision extending to involve the placenta then closure of uterus and finally bilateral internal iliac artery ligation. In conclusion, our experience indicates that conservative methods must be considered to be an option in the management of highly selected cases where fertility is desired. Multi-center studies in tertiary hospitals with large numbers to test this technique.

\section{Conflict of Interest}

The authors report no conflict of interest.

\section{References}

[1] Oyelese, Y. and Smulian, J.C. (2006) Placenta Previa, Placenta Accreta, and Vasa Previa. Obstetrics and Gynecology, 107, 927-941. https://doi.org/10.1097/01.AOG.0000207559.15715.98

[2] Miller, D.A., Chollet, J.A. and Goodwin, T.M. (1997) Clinical Risk Factors for Placenta Previa-Placenta Accreta. American Journal of Obstetrics \& Gynecology, 177, 210-214. https://doi.org/10.1016/S0002-9378(97)70463-0

[3] Wu, S., Kocherginsky, M. and Hibbard, J.U. (2005) Abnormal Placentation: Twenty-Year Analysis. American Journal of Obstetrics \& Gynecology, 192, 1458-1461. https://doi.org/10.1016/j.ajog.2004.12.074

[4] Laban, M., Ibrahim, E.A., Elsafty, M.S. and Hassanin, A.S. (2014) Placenta Accreta Is Associated with Decreased Decidual Natural Killer Cells Population: A Comparative Pilot Study. European Journal of Obstetrics \& Gynecology and Reproductive Biology, 181, 184-188.

[5] Tong, S.Y.P., Tay, K.H. and Kwek, Y.C.K. (2008) Conservative Management of Placenta Accreta: Review of Three Cases. Singapore Medical Journal, 49, 156-159.

[6] Timmermans, S., Van Hof, A.C. and Duvekot, J.J. (2007) Conservative Management of Abnormally Invasive Placentation. Obstetrical and Gynecological Survey, 62, 529-539. https://doi.org/10.1097/01.ogx.0000271133.27011.05

[7] Kayem, G., Davy, C., Goffinet, F., Thomas, C., Clément, D. and Cabrol, D. (2004) Conservative versus Extirpative Management in Cases of Placenta Accreta. Obstetrics and Gynecology, 104, 531-536. https://doi.org/10.1097/01.AOG.0000136086.78099.0f

[8] Hudon, L., Belfort, M.A. and Broome, D.R. (1998) Diagnosis and Management of Placenta Percreta: A Review. Obstetrical \& Gynecological Survey, 53, 509-517. 
https://doi.org/10.1097/00006254-199808000-00024

[9] Gaspare, C., D’Alfonso, A., Armando, N., Giuseppe, R., Valentina, P., et al. (2014) Conservative Surgical Management of Placenta Previa and Accreta. A Case Report. SOJ Surgery, 1, 1-3. https://doi.org/10.15226/2376-4570/1/1/00106

[10] Palacios Jaraquemada, J.M., Pesaresi, M., Nassif, J.C. and Hemosid, S. (2004) Anterior Placenta Percreta Surgical Approach, Hemostasis \& Uterine Repair. Acta $O b$ stetricia et Gynecological Scandinvica, 83, 738-744. https://doi.org/10.1111/j.0001-6349.2004.00517.x

[11] Chandraharan, E., Rao, S., Beli, A.-M. and Arulkumaran, S. (2012) The Triple-P Procedure as a Conservative Surgical Alternative to Peripartum Hysterectomy for Placenta Percreta. International Journal of Gynecology \& Obstetrics, 117, 191-194. https://doi.org/10.1016/j.ijgo.2011.12.005 